\title{
3-D full waveform inversion of seismic data; Part I. Theory
}

Ki Ha Lee*, Ernest Orlando Lawrence Berkeley National Laboratory, USA, KHLee@lbl.gov

\section{Summary}

Full waveform inversion of seismic data is a challenging subject partly because of the lack of precise knowledge of the source. Since currently available approaches involve some form of approximations to the source, inversion results are subject to the quality and the choice of the source information used. A new full waveform inversion scheme has been introduced (Lee and Kim, 2003) using normalized wavefield for simple two-dimensional (2-D) scalar problems. The method does not require source information, so potential inversion errors due to source estimation may be eliminated. A gather of seismic traces is first Fourier-transformed into the frequency domain and a normalized wavefield is obtained for each trace in the frequency domain. Normalization is done with respect to the frequency response of a reference trace selected from the gather, so the complex-valued normalized wavefield is source-independent and dimensionless. The inversion algorithm minimizes misfits between measured normalized wavefield and numerically computed normalized wavefield. In this paper the full waveform inversion is extended to three-dimensional (3-D) problems.

\section{Introduction}

It is a common practice in seismic industry to estimate subsurface velocity structure by analyzing the traveltimes of the seismic signals. In crosshole and surface-to-borehole applications, typical approaches involve ray tomography (e.g., Peterson et al., 1985; Nolet, 1985, Humphreys and Clayton, 1988; Scales et al., 1988; Vasco, 1991) and more recently Fresnel volume tomography (e.g., Cerveny and Soares, 1992; Vasco et al., 1995). Traveltime tomographies using ray tracing require high-frequency approximation, with maximum resolution on the order of a wavelength (Sheng and Schuster, 2000), or a fraction (5\%) of the well separation in some practical cases. Due to lack of resolution, however, usefulness of ray tomography may be limited if the objective is to better understand the petrophysical and hydrological properties of soils and rocks. Such understanding is important in characterizing petroleum and geothermal reservoirs and in environmental applications of various scales.

An alternative to traveltime tomography is full waveform inversion. Recent studies (e.g., Sen and Stoffa, 1991; Kormendi and Dietrich, 1991; Minkoff and Symes, 1997; Zhou et al., 1997; Plessix and Bork, 1998; Pratt, 1999a, 1999b) suggest that full waveform inversion can provide improved resolution of the velocity and density structures.
Amplitudes and phases of waveforms are sensitive to the petrophysical property of the materials through which the wave propagates. Therefore, full waveform analyses may be used as tools in investigating hydrological and petrophysical properties of the medium. There is, however, one major difficulty to overcome in full waveform inversion. In all field applications, the effective source waveform, the coupling between the source and the medium, and the coupling between the receivers and the medium, are not very well understood. The problem can be alleviated to some extent with a good velocity approximation (Pratt, 1999a), but the measured signals cannot be properly calibrated in general, rendering full waveform inversion technically difficult to apply.

To overcome the above difficulty a simple inversion scheme has been proposed and tested using the normalized wavefield (Lee and Kim, 2003). The approach first transforms seismic data into the frequency domain and a set of normalized wavefield is constructed. The normalized wavefield is independent of the spectrum of the source, so the method allows full waveform inversion without requiring the knowledge of the source signature.

\section{Normalized wavefield}

Let us assume a field survey involving NS source positions and $N G$ receiver positions. For general 3-D problems let us consider three component measurements at each receiver position and three source functions at each source position. It is assumed that measurements are in $x, y$, and $z$, and that the vector composition of each of three source functions is unknown. The measurements may be in the form of pressure, displacement, velocity or acceleration, and are described in general as

$$
\begin{aligned}
\mathbf{D}_{j i}^{d}(t)= & \mathbf{R}_{j}(t) \otimes \mathbf{P}_{j i}^{d}(t) \otimes \mathbf{S}_{i}(t), \\
& j=1,2, \ldots, N G, i=1,2, \ldots, N S,
\end{aligned}
$$

where the superscript $d$ indicates data and $\otimes$ signifies convolution in time. Here $\mathbf{D}_{j i}^{d}(t)$ is the trace recorded at the $j$-th receiver position due to the $i$-th source. The source function $\mathbf{S}_{i}(t)$ includes source system function and the radiation pattern caused by source-medium coupling, and therefore is an effective source. $\mathbf{P}_{j i}^{d}(t)$ is the impulse response of the medium at the $j$-th receiver position due to the $i$-th source. The receiver function $\mathbf{R}_{j}(t)$ includes the effects of the receiver system function and the mediumreceiver coupling. In the following analysis $\mathbf{R}_{j}(t)$ will be ignored by assuming that receiver (geophone) calibration is 


\section{3-D Full Waveform Inversion}

known and that the effect of medium-receiver coupling to data can be ignored in comparison with that corresponding to the source. The impulse response $\mathbf{P}_{j i}^{d}(t)$ is the generalized Green's function solution to the wave equation with an impulse source in time at the $i$-th position.

Fourier transforming equation (1), $\operatorname{FT}\{(\mathbf{D}, \mathbf{P}, \mathbf{S})(\mathrm{t})\} \rightarrow$ $(\mathbf{D}, \mathbf{P}, \mathbf{S})(\omega)$, ignoring $\mathbf{R}_{j}(t)$ factor, we get

$$
\mathbf{D}_{j i}^{d}(\omega)=\mathbf{P}_{j i}^{d}(\omega) \mathbf{S}_{i}(\omega)
$$

The data at the $j$-th receiver position in equation (2) may be written in tensor form:

$$
\begin{aligned}
\mathbf{D}_{j i}^{d}(\omega) & =\left[\mathbf{d}_{a j i}^{d}, \mathbf{d}_{b j i}^{d}, \mathbf{d}_{c j i}^{d}\right](\omega) \\
& =\left[\begin{array}{lll}
d_{1 a j i}^{d} & d_{1 b j i}^{d} & d_{1 c j i}^{d} \\
d_{2 a j i}^{d} & d_{2 b j i}^{d} & d_{2 c j i}^{d} \\
d_{3 a j i}^{d} & d_{3 b j i}^{d} & d_{3 c j i}^{d}
\end{array}\right](\omega)
\end{aligned} .
$$

Here, multiple subscripts ' $l k j i$ ' are used to describe data elements. The last two subscripts ' $j i$ ' indicate receiver $(j=$ $1,2, \ldots, N G)$ and source $(i=1,2, \ldots, N S)$ positions, and the first two subscripts ' $l k$ ' are used to describe Cartesian field components $l=(1,2,3)$ generated by three source functions $k=(a, b, c)$. The three source functions are unknown and each source function may in turn be composed of three Cartesian components; i.e., $\mathbf{s}_{a i}=\left(s_{1 a i}, s_{2 a i}, s_{3 a i}\right)^{T}$. So, the vector source function at the $i$-th position may be described by

$\mathbf{S}_{i}(\omega)=\left[\mathbf{s}_{a i}, \mathbf{s}_{b i}, \mathbf{s}_{c i}\right](\omega)=\left[\begin{array}{ccc}s_{1 a i} & s_{1 b i} & s_{1 c i} \\ s_{2 a i} & s_{2 b i} & s_{2 c i} \\ s_{3 a i} & s_{3 b i} & s_{3 c i}\end{array}\right](\omega)$

The impulse response, or the Green's function, of the medium relating the diagonal impulse sources at the $i$-th transmitter position to the measurements at the $j$-th receiver position may be written as

$$
\begin{aligned}
\mathbf{P}_{j i}^{d}(\omega) & =\left[\mathbf{p}_{1 j i}^{d}, \mathbf{p}_{2 j i}^{d}, \mathbf{p}_{3 j i}^{d}\right](\omega) \\
& =\left[\begin{array}{lll}
p_{11 j i}^{d} & p_{12 j i}^{d} & p_{13 j i}^{d} \\
p_{21 j i}^{d} & p_{22 j i}^{d} & p_{23 j i}^{d} \\
p_{31 j i}^{d} & p_{32 j i}^{d} & p_{33 j i}^{d}
\end{array}\right](\omega)
\end{aligned}
$$

To define the normalized wavefield, a reference point is first selected, say $j=1$. The normalized wavefield $\mathbf{T}_{j i}^{d}$ is defined in such a way that $\mathbf{D}_{j i}^{d}=\mathbf{T}_{j i}^{d} \mathbf{D}_{1 i}^{d}, j=2, \ldots, N G$. It has a property of producing data at the $j$-th receiver position when it is post-multiplied by the data at the reference point. Explicitly,

$$
\begin{aligned}
\mathbf{T}_{j i}^{d}(\omega) & =\mathbf{D}_{j i}^{d}(\omega)\left[\mathbf{D}_{1 i}^{d}(\omega)\right]^{-1} \\
& =\mathbf{P}_{j i}^{d}(\omega) \mathbf{S}_{i}(\omega)\left[\mathbf{S}_{i}(\omega)\right]^{-1}\left[\mathbf{P}_{1 i}^{d}(\omega)\right]^{-1} \\
& =\mathbf{P}_{j i}^{d}(\omega)\left[\mathbf{P}_{1 i}^{d}(\omega)\right]^{-1}=\left[\begin{array}{ccc}
t_{11 j i}^{d} & t_{12 j i}^{d} & t_{13 j i}^{d} \\
t_{21 j i}^{d} & t_{22 j i}^{d} & t_{23 j i}^{d} \\
t_{31 j i}^{d} & t_{32 j i}^{d} & t_{33 j i}^{d}
\end{array}\right](\omega),
\end{aligned}
$$

where the convention of the subscripts for the normalized wavefield is the same as that used for the impulse response. In equation (6) the source function cancels out itself, so the normalized wavefield is defined as the normalized impulse response of the medium; hence the uniqueness of the normalized wavefield. The necessary condition for the source function to cancel out is that the determinant of the source matrix is non-zero. In other words, three source functions need to be linearly independent. Each element of the normalized wavefield is described in terms of individual elementary impulse responses in Appendix A.

\section{Full waveform inversion}

To obtain the numerical solution for the impulse response for wave equations one needs to spatially discretize the constitutive parameters, and apply finite difference, finite element, or integral equation technique to solve the discretized system. Using the finite difference or finite element method, the assembled system of equations, including the damping, takes a general form (Marfurt, 1984)

$$
\mathbf{M} \ddot{\mathbf{p}}(t)+\mathbf{C} \dot{\mathbf{p}}(t)+\mathbf{K} \mathbf{p}(t)=\mathbf{g} \delta(t),
$$

where the field vector $\mathbf{p}(t)$ is the discretized wavefield in the time domain, $\mathbf{M}$ is the mass matrix, $\mathbf{K}$ is the stiffness matrix, and $\mathbf{C}$ is the damping matrix. If there is a total of $N$ unknowns in the distretization, all $\mathbf{M}, \mathbf{C}$, and $\mathbf{K}$ are $N \times N$ square matrices, the field vector $\mathbf{p}$ is $N \times 1$, and the load vector $\mathbf{g}$ is also $N \times 1$ whose entries are all zero except a 1 at the node where the source is located. $\delta(t)$ is the Dirac delta function in time, so the wavefield $\mathbf{p}(t)$ is the impulse response. Boundary conditions between adjoining elements are implicitly included (Marfurt, 1984).

The reduced system of equations may be solved in the time domain, typically using the coupled first-order differential equations on a staggered grid (Virieux, 1984 and 1986; Levander, 1988; Randall, 1989; Graves, 1996), or in the frequency domain (Pratt, 1990; Pratt and Worthington; 1990; Song and Williamson, 1995; Song et al., 1995; Pratt et al., 1998) after Fourier transforming equation (7), $F T\{\mathbf{p}(\mathrm{t})\} \rightarrow \mathbf{p}(\omega)$, into

$$
-\omega^{2} \mathbf{M p}(\omega)+i \omega \mathbf{C p}(\omega)+\mathbf{K} \mathbf{p}(\omega)=\boldsymbol{g}
$$




\section{3-D Full Waveform Inversion}

Next, I will show that the normalized wavefield defined by equation (6) is all that's needed for the full waveform inversion. In the inversion the objective functional consists of data misfit, and the misfit in normalized wavefield can be used just for that purpose. For a given model one can generate synthetic data using equation (8), and then obtain the normalized wavefield for the model similar to the normalized wavefield for the data described by equation (6). Formally, the frequency-domain synthetic impulse response at the $j$-th receiver position due to the impulse sources at the $i$-th source position may be obtained and designated as $\mathbf{P}_{j i}^{m}(\omega)$,

$$
\mathbf{P}_{j i}^{m}(\omega)=\left[\begin{array}{ccc}
p_{11 j i}^{m} & p_{12 j i}^{m} & p_{13 j i}^{m} \\
p_{21 j i}^{m} & p_{22 j i}^{m} & p_{23 j i}^{m} \\
p_{31 j i}^{m} & p_{32 j i}^{m} & p_{33 j i}^{m}
\end{array}\right](\omega)
$$

where the superscript $m$ indicates model. Accordingly, the model normalized wavefield is obtained from the numerical solution for the given velocity model

$$
\begin{aligned}
\mathbf{T}_{j i}^{m}(\omega) & =\mathbf{P}_{j i}^{m}(\omega)\left[\mathbf{P}_{1 i}^{m}(\omega)\right]^{-1} \\
& =\left[\begin{array}{lll}
t_{11 j i}^{m} & t_{12 j i}^{m} & t_{13 j i}^{m} \\
t_{21 j i}^{m} & t_{22 j i}^{m} & t_{23 j i}^{m} \\
t_{31 j i}^{m} & t_{32 j i}^{m} & t_{33 j i}^{m}
\end{array}\right](\omega)
\end{aligned}
$$

The inversion procedure starts with the misfit, with the subscripts to the normalized wavefields dropped

$$
\phi(\mathbf{m})=\left\|\mathbf{W}_{d}\left(\mathbf{T}^{m}-\mathbf{T}^{d}\right)\right\|^{2}
$$

where $\|\bullet\|^{2}$ denotes the Euclidean norm and $\mathbf{W}_{d}$ is used to account for the correlated measurement errors in the data. For data with uncorrelated errors $\mathbf{W}_{d}$ is diagonal with the inverse of measurement errors, usually the standard deviation, on its diagonal. The misfit between data and model normalized wavefields at the reference point is always zero $\left(t_{l k 1 i}^{m}-t_{l k 1 i}^{d}=0.0\right)$. In setting up the data misfit, real and imaginary parts are separated, so the actual number of data used for the inversion is $N E Q=2 \times N F R E Q$ $\times N S \times(N G-1) \times 9$, and the computation is done in real arithmetic. The variable NFREQ is the number of frequencies used for the inversion. The number of data includes nine normalized wavefield elements for each source-receiver pair. $\mathbf{W}_{d}$ is an $N E Q \times N E Q$ square matrix, and the data misfit $\left(\mathbf{T}^{m}-\mathbf{T}^{d}\right)$ is an $N E Q \times 1$ column matrix.

For inversion one may consider the Gauss-Newton method by first expanding the objective functional, equation (11), into a Taylor series (e.g., Bertsekas, 1982; Tarantola, 1987; Oldenburg et al., 1993)

$$
\begin{aligned}
& \phi(\mathbf{m}+\delta \mathbf{m})= \\
& \quad \phi(\mathbf{m})+\boldsymbol{\gamma}_{m}^{T} \delta \mathbf{m}+0.5 \delta \mathbf{m}^{T} \mathbf{H}_{m} \delta \mathbf{m}+O\left\{(\delta \mathbf{m})^{3}\right\}
\end{aligned}
$$

Here, $\delta \mathbf{m}$ is a perturbation to the model parameter $\mathbf{m}$, $\gamma_{m}$ is an $M \times 1$ column matrix consisting of elements $\left\{\frac{\partial \phi}{\partial m_{q}}, q=1,2, \ldots, M\right\}$ with $M$ being the total number of parameters to be determined, and is compactly written as

$\gamma_{m}=2 \mathbf{J}^{T} \mathbf{W}_{d}{ }^{T} \mathbf{W}_{d}\left(\mathbf{T}^{m}-\mathbf{T}^{d}\right)$,

where $\mathbf{J}$ is the Jacobian (sensitivity) matrix. $\mathbf{H}_{m}$ is an $M \times$ $M$ square (Hessian) matrix consisting of elements $\left\{\frac{\partial^{2} \phi}{\partial m_{q} \partial m_{r}}, q, r=1,2, \ldots, M\right\}$ written as

$\mathbf{H}_{m}=2 \mathbf{J}^{T} \mathbf{W}_{d}^{T} \mathbf{W}_{d} \mathbf{J}+O\left(\frac{\partial \boldsymbol{J}}{\partial \mathbf{m}}\right)$.

The last term is small if either the residuals are small, or the forward differential equation is quasi linear (Tarantola, 1987). The term is usually difficult to compute and is generally ignored.

The functional that will be minimized consists of the misfit, equation (12), and a constraint that will have a smoothing effect on the variation of the model in the updating process. Specifically, it may be written

$$
\Phi(\mathbf{m}+\delta \mathbf{m})=\phi(\mathbf{m}+\delta \mathbf{m})+\lambda\left\|\mathbf{W}_{m} \delta \mathbf{m}\right\|^{2},
$$

where $\lambda$ is the Lagrange multiplier that controls relative importance of data misfit and model roughness, and $\mathbf{W}_{m}$ is an $M \times M$ weighting matrix of the model parameters. Minimization of functional (15) with respect to perturbation in model parameter results in a system of normal equations

$$
\begin{aligned}
\left(\mathbf{J}^{T} \mathbf{W}_{d}{ }^{T} \mathbf{W}_{d} \mathbf{J}\right. & \left.+\lambda \mathbf{W}_{m}{ }^{T} \mathbf{W}_{m}\right) \delta m \\
& =-\mathbf{J}^{T} \mathbf{W}_{d}{ }^{T} \mathbf{W}_{d}\left(\mathbf{T}^{m}-\mathbf{T}^{d}\right),
\end{aligned}
$$

from which the model parameter at the $(k+1)$-th iteration is updated to

$m_{q}^{k+1}=m_{q}^{k}+\delta m_{q}^{k}, \quad q=1,2, \ldots, M$.

The iteration stops when the change in model parameters is below a preset tolerance, typically given in terms of rootmean-square (rms) misfit. 


\section{3-D Full Waveform Inversion}

\section{Conclusions}

Normalized wavefield depends only on the subsurface model and the position of the source, and is independent of the source spectrum by construction. The highlight of this paper is that 3-D full waveform inversion of seismic data can be achieved using the normalized wavefield, and that with the proposed method potential inversion errors due to source estimation required by conventional full waveform inversion methods can be eliminated.

\section{Acknowledgments}

This work was supported by the Office of Basic Energy Sciences, Engineering and Geosciences Division of the U.S. Department of Energy under Contract No. DE-AC0376SF00098. I thank Frank Morrison for his encouragement of this work at the earlier stage.

\section{References}

Bertsekas, D. P., 1982, Enlarging the region of convergence of Newton's method for constrained optimization: J. Optimization Theory Applications, 36, 221-251.

Cerveny, V., and Soares, J.E.P., 1992, Fresnel volume ray tracing: Geophysics, 57, 902-915.

Graves, R. W., 1996, Simulating seismic wave propagation in 3D elastic media using staggered-gris finite differences: Bull. Seism. Soc. Am., 86, 1091-1106.

Humphreys, E., and Clayton, R. W., 1988, Application of back-projection tomography to seismic traveltime problems: J. Geophys. Res., 93, 1073-1085.

Kormendi, F., and Dietrich, M., 1991, Nonlinear waveform inversion of plane-wave seismograms in stratified elastic media: Geophysics, 56, 664-674.

Lee, K.H., and Kim, H.J., 2003, Source-independent full waveform inversion of seismic data, accepted for publication in Geophysics, LBNL-49934.

Levander, A. R., 1988, Fourth-order finite difference P-SV seismograms: Geophysics, 53, 1425-1436.

Marfurt, K. J., 1984, Accuracy of finite-difference and finite-element modeling of the scalar and elastic wave equations: Geophysics, 49, 533-549.

Minkoff, S. E., and Symes, W. W., 1997, Full waveform inversion of marine reflection data in the plane-wave domain: Geophysics, 62, 540-553.

Nolet, G., 1985, Solving or resolving inadequate and noisy tomographic systems: J. Comp. Phys., 61, 463-482.

Oldenburg, D. W., McGillivray, P. R., and Ellis, R. G., 1993, Generalized subspace methods for large-scale inverse problems: Geophys. J. Int., 114, 12-20.

Peterson, J. E., Paulson, B. N. P., and McEvilly, T. V., 1985, Applications of algebraic reconstruction techniques to crosshole seismic data: Geophysics, 50, 1566-1580.

Plessix, R.- E., and Bork, J., 1998, A full waveform inversion example in VTI media: 68th Ann. Internat.
Mtg., Soc. Expl. Geophys., Expanded Abstracts, 15621565.

Pratt, R. G., 1990, Inverse theory applied to multi-source cross-hole tomography, Part II: elastic wave-equation method: Geophys. Prosp., 38, 311-330.

1999a, Seismic waveform inversion in frequency domain, Part 1: Theory and verification in physical scale model: Geophysics, 64, 888-901.

- 1999b, Seismic waveform inversion in frequency domain, Part 2: Fault delineation in sediments using crosshole data: Geophysics, 64, 902-914.

Pratt, R. G., Shin, C., Hicks, G. J., 1998, Gauss-Newton and full Newton methods in frequency-space seismic waveform inversion: Geophys. J. Int., 133, 341-362.

Pratt, R. G., and Worthington, M. H., 1990, Inverse theory applied to multi-source cross-hole tomography, Part I: acoustic wave-equation method: Geophys. Prosp., 38, 287-310.

Randall, C. J., 1989, Absorbing boundary condition for the elastic wave equation, velocity-stress formulation: Geophysics, 54, 1141-1152.

Scales, J. A., Gersztenkorn, A., and Treitel, S., 1988, Fast solution of large sparce, linear systems: Application to seismic traveltime tomography: J. Comp. Phys., 75, 314333.S

Sen, M. K., and Stoffa, P. L., 1991, Nonlonear onedimensional seismic waveform inversion using simulated annealing: Geophysics, 56, 1624-1638.

Sheng, J., and Schuster, G. T., 2000, Finite-frequency resolution limits of traveltime tomography for smoothly varying velocity models: 70th Ann. Internat. Mtg., Soc. Expl. Geophys., Expanded Abstracts, 2134-2137.

Song, Z.- M., and Williamson, P. R., 1995, Frequencydomain acoustic modeling and inversion of crosshole data: part I-2.5-D modeling method: Geophysics, 60, 784-795.

Song, Z.- M., Williamson, P. R., and Pratt, R. G., 1995, Frequency-domain acoustic wave modeling and inversion of crosshole data: Part II-inversion method, synthetic experiments and real-data results: Geophysics, 60, 796-809.

Vasco, D. W., 1991, Bounding seismic velocities using a tomographic method: Geophysics, 56, 472-482.

Vasco, D.W., Peterson, Jr., J.E., and Majer, E.L., 1995, Beyond ray tomography: Wavepaths and Fresnel volumes: Geophysics, 60, 1790-1804.

Virieux, J., 1984, P-SV wave propagation in heterogeneous media: velocity-stress finite-difference method: Geophysics, 51, 889-901.

Virieux, J., 1986, SH wave propagation in heterogeneous media: velocity-stress finite-difference method: Geophysics, 49, 1933-1957.

Tarantola, A., 1987, Inverse Problem Theory: Methods for Data Fitting and Parameter Estimation: Elsevier, Amsterdam.

Zhou, C., Schuster, G. T., Hassanzadeh, S., and Harris, J. M, 1997, Elastic wave equation traveltime and wavefield inversion of crosswell data: Geophysics, 62, 853-868. 